BMJ Open

Sport \&

Exercise

Medicine

\title{
Association between physical activity, cardiorespiratory fitness and clustered cardiovascular risk in South African children from disadvantaged communities: results from a cross-sectional study
}

Ivan Müller (D), ${ }^{1}$ Cheryl Walter (D), ${ }^{2}$ Rosa Du Randt (D), ${ }^{2}$ Ann Aerts (D), ${ }^{3}$ Larissa Adams (D), ${ }^{2}$ Jan Degen (D), ${ }^{1}$ Stefanie Gall (D), ${ }^{1}$ Nandi Joubert (D), ${ }^{4,5}$ Siphesihle Nqweniso (D), ${ }^{2}$ Sarah Des Rosiers, ${ }^{3}$ Danielle Smith (D), ${ }^{2}$ Harald Seelig (D), ${ }^{1}$ Peter Steinmann (D), ${ }^{4,5}$ Christina Wadhwani (D), ${ }^{6}$ Nicole Probst-Hensch (iD, ${ }^{4,5}$ Jürg Utzinger (D), ${ }^{4,5}$ Uwe Pühse (D), ${ }^{1}$ Markus Gerber (D) ${ }^{1}$

To cite: Müller I, Walter C, Du Randt $\mathrm{R}$, et al. Association between physical activity, cardiorespiratory fitness and clustered cardiovascular risk in South African children from disadvantaged communities: results from a cross-sectional study. BMJ Open Sport \& Exercise Medicine 2020;0: e000823. doi:10.1136/ bmjsem-2020-000823

Accepted 25 August 2020
Check for updates

(c) Author(s) (or their employer(s)) 2020. Re-use permitted under CC BY-NC. No commercial re-use. See rights and permissions. Published by BMJ.

For numbered affiliations see end of article.

Correspondence to

Ivan Müller;

ivan.mueller@unibas.ch

\section{ABSTRACT}

Background/Aim Physical inactivity (PIA) is a growing global health problem and evidence suggests that PIA is a key driver for cardiovascular and chronic diseases. Recent data from South Africa revealed that only about half of the children achieved recommended daily physical activity (PA) levels. Assessing the intensity of PA in children from low socioeconomic communities in low-income and middle-income countries is important to estimate the extent of cardiovascular risk and overall impact on health.

Methods We conducted a cross-sectional survey in eight quintile 3 primary schools in disadvantaged communities in the Port Elizabeth region, South Africa. Children aged 10-15 years were subjected to PA, blood pressure, cholesterol, blood glucose and skinfold thickness assessments. Cardiovascular risk markers were converted into standardised z-scores and summed, to obtain a clustered cardiovascular risk score.

Results 0verall, 650 children had complete data records. $40.8 \%$ of the children did not meet recommended PA levels (ie, logged $<60$ min of moderate-to-vigorous physical activity (MVPA) per day). If quartiles were developed based on children's cardiorespiratory fitness (CRF) and MVPA levels, a significant difference was found in clustered cardiovascular risk among children in the highest versus lowest fitness $(p<0.001)$ or MVPA $(p<0.001)$ quartiles.

Conclusions CRF and objectively assessed PA are closely linked with children's clustered cardiovascular risk. Given that 4 out of 10 South African schoolchildren from marginalised communities do not meet international PA recommendations, efforts should be made to ensure that promoting a physically active lifestyle is recognised as an important educational goal in primary schools.

Trial registration numbers ISRCTN68411960 and H14HEA-HMS-002.

\section{What are the new findings?}

- Here, we present physical activity and cardiorespiratory fitness profiles among 10-year-old to 15-year-old children from disadvantaged communities in South Africa.

- We found that when quartiles were formed based on children's cardiorespiratory fitness or moderate-tovigorous physical activity levels, there was a clear gradient of decreased clustered cardiovascular risk among children with higher fitness or higher moderate-to-vigorous physical activity levels.

How might it impact on clinical practice in the future?

The findings of our study emphasise the importance of a physically active lifestyle and call for action to address these issues in underserved communities and schools such as those in the townships of Port Elizabeth

- There is a pressing need to design policies and create environments that allow children to achieve international physical activity recommendations. Since the cardiovascular risk of children living in marginalised communities depends on various lifestyle factors, longitudinal and comprehensive monitoring is also required.

\section{INTRODUCTION}

Sedentary lifestyle and physical inactivity (PIA) are growing global health issues and evidence suggests that PIA is a key driver for cardiovascular and chronic diseases. ${ }^{1}$ At a global scale, the prevalence of obesity 
increased at an alarming rate from $0.9 \%$ in girls and $0.7 \%$ in boys in 1975 to $7.8 \%$ in girls and $5.6 \%$ in boys in $2016 .{ }^{2}$ Recognising the negative public health consequences of a sedentary lifestyle encouraged new research to investigate and promote public health interventions to improve physical activity (PA) ${ }^{3-6}$ The first PA guidelines for children were published in $1988 .{ }^{7}$ At present, children aged 5-17 years are advised to pursue moderate-to-vigorous physical activity (MVPA) for at least $60 \mathrm{~min}$ per day. ${ }^{8}$ The current and projected PA levels for low socioeconomic status (SES) communities are worse in lowincome and middle-income countries (LMICs), compared to more affluent populations in LMICs. ${ }^{9} 10$

In low socioeconomic communities in South Africa, unsafe neighbourhoods and poor school facilities typically offer children no or only limited opportunities to play and be active. ${ }^{11}$ Hence, we hypothesise that a considerable portion of South African children from poor neighbourhoods will not meet the recommended PA guidelines. For instance, in 2018, only around half of South African children were reported to have achieved daily PA recommendations. ${ }^{12}$ In order to estimate the health impacts of insufficient PA in children from lower socioeconomic communities, it is necessary to examine their cardiovascular disease risk factors. ${ }^{13}$

We designed a cross-sectional study in the Port Elizabeth region in South Africa with the following aims. First, we described the cardiovascular health risk, PA behaviour and cardiorespiratory fitness (CRF) levels of children attending primary schools in lower socioeconomic communities. Second, we examined differences in these variables dependent on children's age, sex, SES and ethnicity. Third, we investigated whether PA and CRF are independently associated with a composite measure of children's cardiovascular health risk.

\section{METHODS}

\section{Study setting}

The study was conducted in eight quintile 3 primary schools located in disadvantaged communities in and around Port Elizabeth, South Africa. It should be noted that South African schools are ranked into five quintile groups in accordance with the national poverty table, and based on the income levels, dependency ratios and literacy rates in a specific area. ${ }^{14}$ The procedures to select the schools for this study have been described in the study protocol. ${ }^{15}$

\section{Participants' involvement and procedures}

The current investigation is based on cross-sectional data from the 2-year follow-up of the 'Disease, Activity and Schoolchildren's Health' (DASH) study. DASH, a clusterrandomised controlled trial, implemented different school-based health interventions (ie, PA promotion, health education, deworming and nutritional supplementation) over a period of 20 weeks in order to improve children's health status. Children were eligible for inclusion if they assented, did not simultaneously participate in other clinical trials during the study period and did not suffer from medical conditions (as determined by qualified clinical personnel) that prevented participation. Written informed consent was obtained from parents/ legal guardians before the first data assessment and after sharing detailed written and oral information about the study's aims, procedures, risks and benefits. The study protocol was approved by the responsible ethics committees in South Africa and Switzerland. Approval was also obtained from South Africa's Eastern Cape Provincial Departments of Education and Health.

Participants were not directly involved in the design of the study presented here. However, the development of the research questions, study procedures and outcome measures were informed by participants' experience, needs and preferences, as expressed in the formal meetings arranged with education authorities, where these matters were discussed. Caregivers (eg, parents and guardians of participating children) and lay people (eg, education authorities) were involved in the recruitment to and conduct of the study. Recruitment commenced in September 2014, baseline assessment was done in February 2015 and 2-year follow-up data were obtained in February and March 2018. At baseline, all children $(n=1009)$ were in grade 4 and their age ranged between 8 and 13 years. The present study included 650 children who had complete data records at follow-up; hence, the children's age ranged from 10 to 15 years. At school level, our research team, together with the Department of Health, disseminated the main findings.

\section{Measures}

Blood pressure was assessed with a validated oscillometric digital blood pressure monitor (Omron M6 AC, Hoofddorp, Netherlands). Three measurements were performed, and the mean of the last two measurements were employed as indicators of systolic and diastolic blood pressure. Using a Harpenden skinfold calliper (Baty International, West Sussex, UK), skinfold thickness was assessed three times at triceps and subscapular skinfold sites. The mean of all six measurements was taken as the overall skinfold thickness index. ${ }^{16}$ Capillary blood sampling was used to assess blood markers for cardiovascular risk, using the Alere Afinion AS100 analyser (Abbott Technologies, Abbott Park, Illinois, USA). Total cholesterol, high-density lipoprotein (HDL) cholesterol, lowdensity lipoprotein (LDL) cholesterol, triglycerides and blood glucose (HbA1c) were assessed using fasting capillary blood samples. ${ }^{17}$ Evidence of the clinical utility and accuracy of this fingerstick technology has been described previously. $^{18}$

To objectively assess children's PA, children wore a light triaxial accelerometer device (ActiGraph wGT3XBT, Pensacola, Florida, USA) for 7 consecutive days. Evidence for the validity and reliability of this device has been published. ${ }^{19}$ Children were instructed to wear the accelerometer around the hip, except for activities that involved contact with water. Data were recorded at 
a sample rate of $30 \mathrm{~Hz}$. Analysis was performed with ActiLife software version 6.13.2, using raw data set to epochs of $10 \mathrm{~s}$. Non-wear time was determined using the algorithm defined by Troiano and colleagues. ${ }^{20}$ Children's data were considered valid if the accelerometer was worn for at least 8 hours a day, on 4 weekdays and 1 weekend day. ${ }^{21}{ }^{22}$ Furthermore, data were restricted to the period between 06:00 and midnight, hence 18 hours per day. Using the cut-points for children defined by Evenson, we considered time spent sedentary, and in light physical activity (LPA), moderate physical activity (MPA) and vigorous physical activity (VPA). ${ }^{23}$ MPA and VPA were summed up to generate an overall index for MVPA. For all PA indices, results are reported in $\min /$ day.

Based on a standardised test protocol, children's CRF was assessed with the $20 \mathrm{~m}$ shuttle run test, with a starting pace of $8 \mathrm{~km} / \mathrm{h}$. Based on the number of completed laps, children's maximum oxygen uptake $\left(\mathrm{VO}_{2} \max \right)$ was estimated, using a standard protocol. ${ }^{24}{ }^{25}$ Evidence for the reliability and validity of the $20 \mathrm{~m}$ shuttle run test has been reported. $^{26}$

\section{Statistical analysis}

Descriptive statistics for all study variables are reported for the total sample. In the case of severe non-normality (skewness and kurtosis values of $\geq|2|$ and $\geq|7|$, respectively), measured values were log-transformed (natural $\log$ ) before calculating inferential statistics. ${ }^{27}$ Univariate analyses of variance (ANOVAs) were performed to examine differences based on children's age, sex, SES and ethnicity. $\chi^{2}$ tests were carried out to examine whether based on children's age, sex, SES and ethnicity, some children were over-represented/under-represented in the group that did not meet current PA standards. Additionally, a series of linear regression analyses were performed to determine whether and to what extent PA and CRF might predict the various cardiovascular risk markers. Since ANOVAs revealed significant differences $(p<0.05)$ between schools in most of the study variables, we used mixed linear regression with random intercepts for school classes to account for the nested nature of the data (learners assessed in classes) and for potential influences of class and school on the association between PA and CRF with the cardiovascular risk markers. The regression analyses were further controlled for children's age, sex, SES and ethnicity. To examine the independent contribution of CRF and PA, analyses were controlled for total time spent in MVPA, when CRF was used as a predictor. By contrast, when PA indicators were used to predict cardiovascular risk, the analyses were controlled for CRF. All risk markers were z-standardised and summed up to obtain a clustered cardiovascular risk score. Similar to previous publications based on data of the European Youth Heart Study, ${ }^{28}$ the following formula was used in the present study to compute the clustered risk score: (systolic + diastolic blood pressure/2) + mean of six skinfold measurements $+($ HDL-cholesterol $\times-1)+$
LDL-cholesterol + triglycerides + blood glucose. To examine whether (a) students classified to different CRF and PA quartiles and (b) students who met versus did not meet current PA guidelines differ in clustered cardiovascular risk, three analysis of covariance (ANCOVAs) (controlled for age, sex, SES and ethnicity) were performed. Differences between the different quartile groups were analysed with Bonferroni posthoc tests. All analyses were carried out with SPSS version 24 for Mac. Associations were considered as significant, if resulting $\mathrm{p}$ values were $<0.05$.

\section{RESULTS}

Demographic features of the 650 children who had complete data records are shown in table 1. In short, the sample was composed of 351 girls and 299 boys with a mean age of 12.4 years (SD 0.9 years) at endline. In terms of ethnicity, 384 children were black African, 232 were of coloured ethnicity, while for the remaining 34 children, this information was not available. Although children lived in disadvantaged areas, most of them had relatively high scores on the SES index. Table 1 also provides descriptive statistics for cardiovascular health risk markers, PA behaviour indices and CRF. Overall, $40.8 \%$ of the children did not meet recommended PA levels, therefore achieved $<60 \mathrm{~min}$ MVPA per day.

As shown in table 2, children aged 13-15 years had higher measured systolic and diastolic blood pressure than their younger counterparts aged 10-12 years. While older children engaged less in sedentary activity and LPA, they had higher scores for MPA, VPA, MVPA and CRF. Compared to boys, girls had higher readings for diastolic blood pressure, thickness of skinfolds, triglyceride levels and sedentary activity. Girls also had a higher clustered cardiovascular risk than boys. By contrast, boys had higher scores for HbA1c, LPA, MPA, VPA, MVPA and CRF. Differences between boys and girls were particularly marked with regard to PA behaviour and CRF, where levels of explained variance ranged from $2.1 \%$ to $28.4 \%$. Only small differences were observed with regard to children's SES. Children with higher SES had higher skinfold thickness and performed somewhat more poorly in the CRF test. Ethnic differences appeared in most study variables showing that black African children had higher scores with regard to thickness of skinfolds, sedentary activity and LPA, whereas coloured children had higher scores for diastolic blood pressure, total cholesterol, LDL cholesterol, triglycerides, MPA, VPA, MVPA and CRF. The portion of children not meeting international PA recommendations was higher among younger children $\left(\chi^{2}(1650)=6.0, \mathrm{p}<0.05,44.6 \%\right.$ vs $\left.34.9 \%\right)$, girls $\left(\chi^{2}(1650)\right.$ $=108.0, \mathrm{p}<0.001,59.3 \%$ vs $19.1 \%)$ and black African children $\left(\chi^{2}(1650)=12.3, \mathrm{p}<0.001,47.1 \%\right.$ vs $\left.32.8 \%\right)$.

Table 3 provides information on whether CRF and PA indices are independently associated with cardiovascular health risk markers. After controlling for MVPA, class-inschool (random intercept) and potential confounders 
Table 1 Descriptive characteristics of 650 children from Port Elizabeth, South Africa, in February and March 2018

\begin{tabular}{|c|c|c|c|c|c|c|}
\hline Outcome variables & $\mathbf{M}$ & SD & Min & Max & Skew & Kurt \\
\hline Systolic blood pressure $(\mathrm{mm} \mathrm{Hg})$ & 109.5 & 11.3 & 78.0 & 145.0 & 0.2 & -0.1 \\
\hline Diastolic blood pressure $(\mathrm{mm} \mathrm{Hg})$ & 66.5 & 8.1 & 42.0 & 110.0 & 0.4 & 1.2 \\
\hline Skinfolds (mm) & 11.1 & 6.2 & 3.6 & 40.9 & 1.8 & 3.5 \\
\hline Total cholesterol (mmol/L) & 3.6 & 0.6 & 2.5 & 6.1 & 0.8 & 0.5 \\
\hline HDL cholesterol (mmol/L) & 1.4 & 0.3 & 0.7 & 2.6 & 0.7 & 0.5 \\
\hline LDL cholesterol (mmol/L) & 1.8 & 0.6 & 0.5 & 3.9 & 0.6 & 0.5 \\
\hline Triglycerides (mmol/L) & 0.8 & 0.3 & 0.5 & 3.3 & 2.6 & 11.9 \\
\hline $\mathrm{HbA1c}(\%)$ & 5.5 & 2.3 & 4.4 & 6.4 & -0.4 & 1.7 \\
\hline Clustered risk index & 0.0 & 2.9 & -6.6 & 11.8 & 0.6 & 0.8 \\
\hline \multicolumn{7}{|l|}{ Predictor variables } \\
\hline Sedentary activity (min/day) & 663.3 & 84.6 & 296.8 & 854.0 & -1.0 & 1.9 \\
\hline Light physical activity (min/day) & 261.8 & 50.4 & 51.0 & 409.3 & -0.5 & 1.1 \\
\hline Moderate physical activity (min/day) & 50.8 & 19.5 & 7.6 & 127.9 & 0.6 & 0.4 \\
\hline Vigorous physical activity (min/day) & 21.7 & 15.6 & 1.2 & 117.0 & 1.7 & 4.5 \\
\hline Moderate-to-vigorous physical activity (min/day) & 72.5 & 32.9 & 8.8 & 218.6 & 0.9 & 1.2 \\
\hline Cardiorespiratory fitness (completed laps in $20 \mathrm{~m}$ shuttle run test) $\dagger$ & 36.4 & 20.2 & 6.0 & 99.0 & 0.8 & 0.0 \\
\hline \multicolumn{7}{|l|}{ Potential confounders (metric) } \\
\hline Age (years) & 12.4 & 0.9 & 10.0 & 15.0 & 0.5 & 0.1 \\
\hline Socioeconomic status $(0-9) \ddagger$ & 7.6 & 2.0 & 0.0 & 9.0 & -1.6 & 2.0 \\
\hline Potential confounders (nominal) & $\mathrm{N}$ & $\%$ & & & & \\
\hline \multicolumn{7}{|l|}{ Sex } \\
\hline Girls, n (\%) & 351 & $(54.0)$ & & & & \\
\hline Boys, $n(\%)$ & 299 & $(46.0)$ & & & & \\
\hline \multicolumn{7}{|l|}{ Ethnicity§ } \\
\hline Black African, n (\%) & 384 & (62.3) & & & & \\
\hline Coloured, n (\%) & 232 & $(37.7)$ & & & & \\
\hline
\end{tabular}

†Seventeen children with missing values.

$\ddagger$ Thirty-seven children with missing values.

$\S$ Thirty-four children with missing values.

$\mathrm{N}=596$ after listwise exclusion of missing cases. Scores for triglycerides were log-transformed (skewness (skew) and kurtosis (kurt) values presented in brackets).

HbA1c, blood glucose; HDL, high-density lipoprotein; LDL, low-density lipoprotein.

(age, sex, SES and ethnicity), higher CRF levels were negatively associated with systolic blood pressure, thickness of skinfolds, total cholesterol and LDL cholesterol. A statistically non-significant trend was also observed for diastolic blood pressure. Higher levels of CRF were significantly and negatively associated with the clustered cardiovascular risk index. Similar (although weaker) relationships were observed for MVPA. Thus, after controlling for class-in-school (random intercept), CRF and potential confounders (accelerometer wear-time, age, sex, SES and ethnicity), children who engaged in more MVPA had lower systolic blood pressure, lower thickness of skinfolds and a lower clustered cardiovascular risk. A statistically non-significant trend was also observed for total cholesterol. Furthermore, table 3 shows that children who spent more time in sedentary activities had higher systolic blood pressure, although sedentariness was not significantly associated with any other cardiovascular health risk marker.
Based on ANCOVAs, figure 1 shows that if children's CRF and MVPA levels were based on quartiles, a clear gradient of lower clustered cardiovascular risk among children with higher CRF or MVPA levels could be observed (figure 1A). The same is true if children who met PA recommendations were compared with peers who did not (figure 1B).

\section{DISCUSSION}

Quartiles based on children's CRF or MVPA levels showed evidence of decreased clustered cardiovascular risk among children with higher fitness or MVPA levels living in lower socioeconomic neighbourhoods in Port Elizabeth, South Africa. To our knowledge, objective assessments of PA from lower socioeconomic communities in South Africa, as a representative of a LMIC, have not yet been carried out to the extent and intensity of this study.

Compared to the European Youth Heart Study, we used a slightly different formula to calculate an overall 
Table 2 Differences in outcome and predictor variables, based on children's age, sex, socioeconomic status and ethnicity of 650 children from Port Elizabeth, South Africa, in February and March 2018

\begin{tabular}{|c|c|c|c|c|c|c|c|c|c|c|c|c|}
\hline & \multicolumn{6}{|l|}{ Age } & \multicolumn{6}{|l|}{ Sex } \\
\hline & \multicolumn{2}{|c|}{$10-12$ years } & \multicolumn{2}{|c|}{$13-15$ years } & \multirow[b]{2}{*}{$\mathbf{F}$} & \multirow[b]{2}{*}{$\eta^{2}$} & \multicolumn{2}{|l|}{ Boys } & \multicolumn{2}{|l|}{ Girls } & \multirow[b]{2}{*}{$\mathbf{F}$} & \multirow[b]{2}{*}{$\eta^{2}$} \\
\hline & $\mathbf{M}$ & SD & $\mathbf{M}$ & SD & & & $\mathbf{M}$ & SD & $\mathbf{M}$ & SD & & \\
\hline Systolic blood pressure $(\mathrm{mm} \mathrm{Hg})$ & 108.5 & 11.0 & 111.1 & 11.6 & $8.7^{\star \star}$ & 0.001 & 109.1 & 12.1 & 109.9 & 10.6 & 0.8 & - \\
\hline $\begin{array}{l}\text { Diastolic blood pressure }(\mathrm{mm} \\
\mathrm{Hg})\end{array}$ & 65.9 & 7.8 & 67.5 & 8.4 & $6.4^{\star \star}$ & 0.010 & 65.8 & 8.2 & 67.2 & 8.0 & $4.5^{*}$ & 0.007 \\
\hline Skinfolds (mm) & 11.4 & 6.2 & 10.6 & 6.1 & 2.8 & - & 8.9 & 5.2 & 13.0 & 6.3 & $81.1^{\star \star \star}$ & 0.111 \\
\hline Total cholesterol $(\mathrm{mmol} / \mathrm{L})$ & 3.6 & 0.7 & 3.5 & 0.6 & 2.4 & - & 3.5 & 0.6 & 3.6 & 0.7 & 3.8 & - \\
\hline HDL cholesterol (mmol/L) & 1.4 & 0.3 & 1.4 & 0.3 & 2.2 & - & 1.4 & 0.4 & 1.4 & 0.3 & 0.0 & - \\
\hline LDL cholesterol (mmol/L) & 1.8 & 0.5 & 1.8 & 0.6 & 0.9 & - & 1.7 & 0.5 & 1.8 & 0.6 & 3.0 & - \\
\hline Triglycerides (mmol/L) & 0.8 & 0.3 & 0.8 & 0.3 & 0.0 & - & 0.8 & 0.3 & 0.8 & 0.3 & $7.2^{\star \star}$ & 0.011 \\
\hline $\mathrm{HbA1c}(\%)$ & 5.5 & 0.2 & 5.5 & 0.3 & 2.5 & - & 5.5 & 0.2 & 5.5 & 0.2 & $8.3^{\star \star}$ & 0.013 \\
\hline Clustered risk index & 0.0 & 2.8 & 0.0 & 3.1 & 0.2 & - & -0.5 & 2.9 & 0.4 & 2.8 & $16.4^{\star \star *}$ & 0.025 \\
\hline Sedentary activity & 670.5 & 77.8 & 652.3 & 93.4 & $7.2^{\star \star}$ & 0.011 & 643.4 & 88.5 & 680.4 & 77.3 & $32.4^{\star \star \star}$ & 0.048 \\
\hline Light physical activity (min/day) & 266.8 & 46.3 & 254.2 & 55.4 & $9.9^{* \star}$ & 0.015 & 269.8 & 47.8 & 255.1 & 51.6 & $14.0^{\star \star \star}$ & 0.021 \\
\hline $\begin{array}{l}\text { Moderate physical activity } \\
\text { (min/day) }\end{array}$ & 48.5 & 19.2 & 54.2 & 19.4 & $13.6^{\star \star \star}$ & 0.021 & 60.5 & 18.7 & 42.5 & 16.0 & $174.9^{\star \star \star}$ & 0.213 \\
\hline $\begin{array}{l}\text { Vigorous physical activity } \\
\text { (min/day) }\end{array}$ & 20.8 & 14.9 & 23.3 & 16.5 & $3.9^{*}$ & 0.006 & 30.5 & 17.5 & 14.4 & 8.3 & $235.1^{* * *}$ & 0.266 \\
\hline $\begin{array}{l}\text { Moderate-to-vigorous physical } \\
\text { activity (min/day) }\end{array}$ & 69.3 & 32.3 & 77.5 & 33.4 & $9.7^{* \star}$ & 0.015 & 91.0 & 33.5 & 56.8 & 22.8 & $236.2^{\star \star \star}$ & 0.266 \\
\hline \multirow[t]{4}{*}{$\begin{array}{l}\text { Cardiorespiratory fitness (laps in } \\
20 \text { m shuttle run test) }\end{array}$} & 33.3 & 18.7 & 41.1 & 21.7 & $23.2^{\star \star \star}$ & 0.036 & 48.0 & 20.2 & 26.4 & 14.0 & $250.7^{\star \star \star}$ & 0.284 \\
\hline & \multicolumn{6}{|c|}{ Socioeconomic status $\dagger$} & \multirow{2}{*}{\multicolumn{2}{|c|}{ Ethnicity $\ddagger$}} & & & & \multirow[b]{3}{*}{$\eta^{2}$} \\
\hline & \multicolumn{2}{|c|}{ Lower (0-7) } & \multicolumn{2}{|c|}{ Higher (8-9) } & \multirow[b]{2}{*}{$\mathrm{F}$} & \multirow[b]{2}{*}{$\eta^{2}$} & & & \multicolumn{2}{|c|}{ Coloured } & & \\
\hline & M & SD & M & SD & & & M & SD & \multicolumn{2}{|c|}{$\mathrm{M} \quad \mathrm{S}$} & $\mathrm{F}$ & \\
\hline Systolic blood pressure (mm Hg) & 109.7 & 11.3 & 109.3 & 11.4 & 0.2 & - & 108.8 & 11.6 & 110.3 & 10.8 & 2.7 & - \\
\hline $\begin{array}{l}\text { Diastolic blood pressure } \\
(\mathrm{mm} \mathrm{Hg})\end{array}$ & 66.4 & 8.0 & 66.6 & 8.2 & 0.1 & - & 65.4 & 8.4 & 68.4 & 7.4 & $20.7^{\star \star *}$ & 0.033 \\
\hline Skinfolds (mm) & 10.0 & 5.3 & 11.7 & 6.6 & $9.1^{\star *}$ & 0.015 & 11.9 & 6.5 & 10.0 & 5.7 & 13.7 & 0.022 \\
\hline Total cholesterol (mmol/L) & 3.6 & 0.6 & 3.5 & 0.7 & 1.5 & - & 3.5 & 0.6 & 3.7 & 0.7 & $12.7^{\star \star \star}$ & 0.020 \\
\hline HDL cholesterol (mmol/L) & 1.4 & 0.3 & 1.4 & 0.3 & 1.1 & - & 1.4 & 0.3 & 1.4 & 0.3 & 2.6 & - \\
\hline LDL cholesterol (mmol/L) & 1.8 & 0.5 & 1.8 & 0.6 & 2.7 & - & 1.7 & 0.5 & 1.8 & 0.6 & $4.8^{*}$ & 0.008 \\
\hline Triglycerides (mmol/L) & 0.8 & 0.3 & 0.8 & 0.3 & 2.2 & - & 0.8 & 0.3 & 0.9 & 0.3 & $15.6^{\star *}$ & 0.025 \\
\hline $\mathrm{HbA1c}(\%)$ & 5.5 & 0.2 & 5.5 & 0.2 & 0.3 & - & 5.5 & 0.2 & 5.5 & 0.2 & 3.0 & - \\
\hline Clustered risk index & 0.0 & 2.8 & -0.2 & 3.0 & 0.1 & - & -0.1 & 3.0 & 0.1 & 2.9 & 0.6 & - \\
\hline Sedentary activity & 662.3 & 84.4 & 665.5 & 83.4 & 0.2 & - & 676.6 & 78.2 & 643.7 & 88.3 & $23.2^{\star \star \star}$ & 0.036 \\
\hline Light physical activity (min/day) & 259.8 & 49.0 & 261.8 & 51.3 & 0.2 & - & 268.5 & 46.1 & 250.0 & 55.7 & $19.8^{\star * \star}$ & 0.031 \\
\hline $\begin{array}{l}\text { Moderate physical activity } \\
\text { (min/day) }\end{array}$ & 51.4 & 19.5 & 49.7 & 19.2 & 1.1 & - & 47.8 & 18.1 & 54.9 & 20.8 & $19.9^{\star \star \star}$ & 0.031 \\
\hline $\begin{array}{l}\text { Vigorous physical activity } \\
\text { (min/day) }\end{array}$ & 21.3 & 15.0 & 21.6 & 15.9 & 0.1 & - & 20.1 & 14.6 & 23.9 & 17.0 & $8.5^{\star \star}$ & 0.014 \\
\hline $\begin{array}{l}\text { Moderate-to-vigorous physical } \\
\text { activity (min/day) }\end{array}$ & 72.7 & 32.2 & 71.3 & 33.0 & 0.2 & - & 67.9 & 30.7 & 78.7 & 35.3 & $16.2^{\star \star \star}$ & 0.026 \\
\hline $\begin{array}{l}\text { Cardiorespiratory fitness (laps in } \\
20 \text { m shuttle run test) }\end{array}$ & 40.0 & 20.7 & 35.0 & 20.1 & $7.8^{\star \star}$ & 0.013 & 33.5 & 19.5 & 41.7 & 20.8 & $23.0^{\star \star \star}$ & 0.037 \\
\hline
\end{tabular}

$\dagger \mathrm{N}=37$ participants had missing values for SES.

$\ddagger \mathrm{N}=34$ participants had missing values for ethnicity.

${ }^{*} \mathrm{p}<0.05,{ }^{* *} \mathrm{p}<0.01,{ }^{* * *} \mathrm{p}<0.001$.

Age: 10-12 years $(n=395), 13-15$ years $(n=255)$; sex: boys $(n=299)$, girls $(n=351)$; socioeconomic status: lower $(n=194)$, higher $(n=419)$; ethnicity: black African $(n=384)$, coloured $(n=232)$.

HbA1c, blood glucose; HDL, high-density lipoprotein; LDL, low-density lipoprotein. 


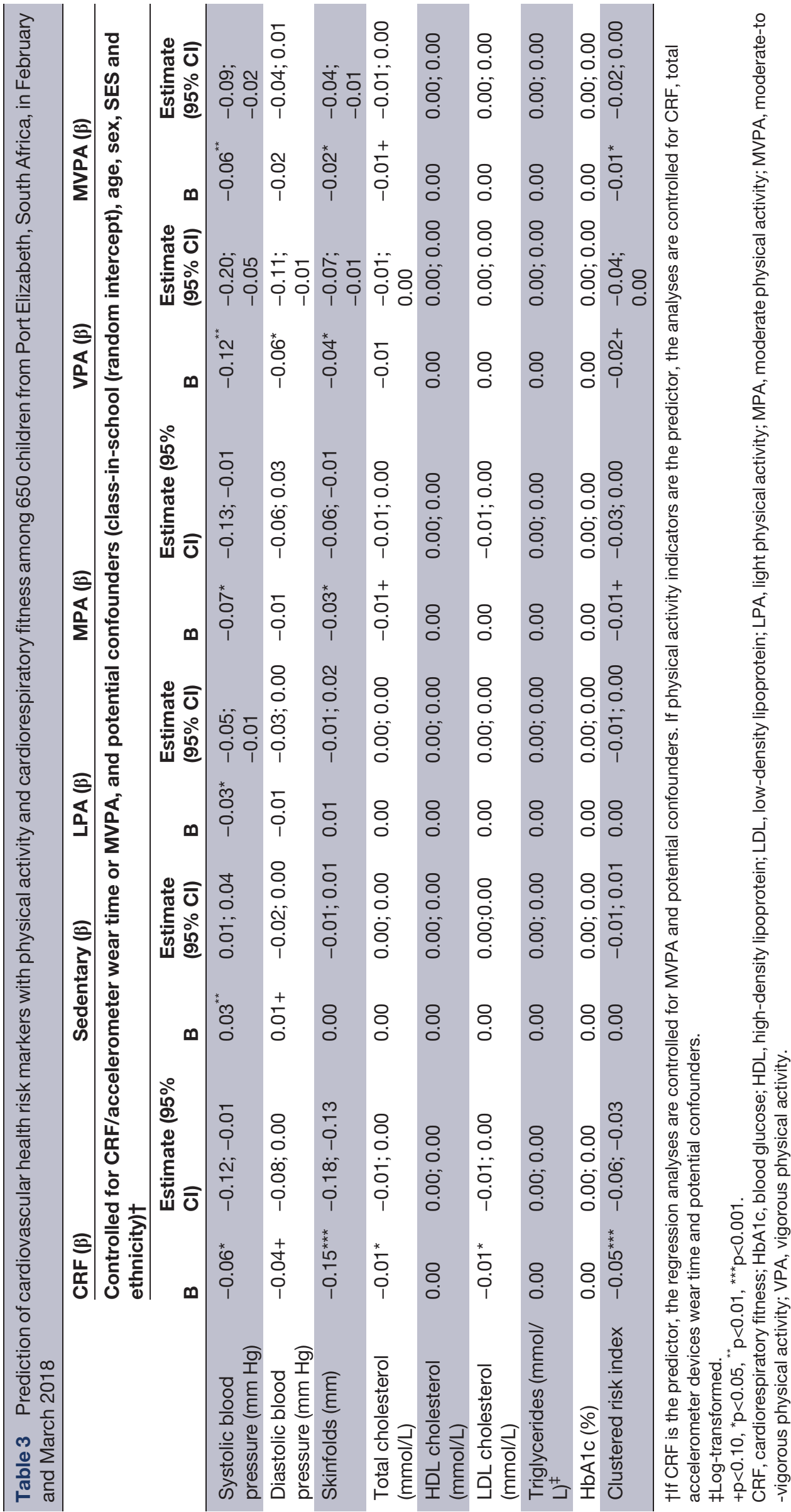



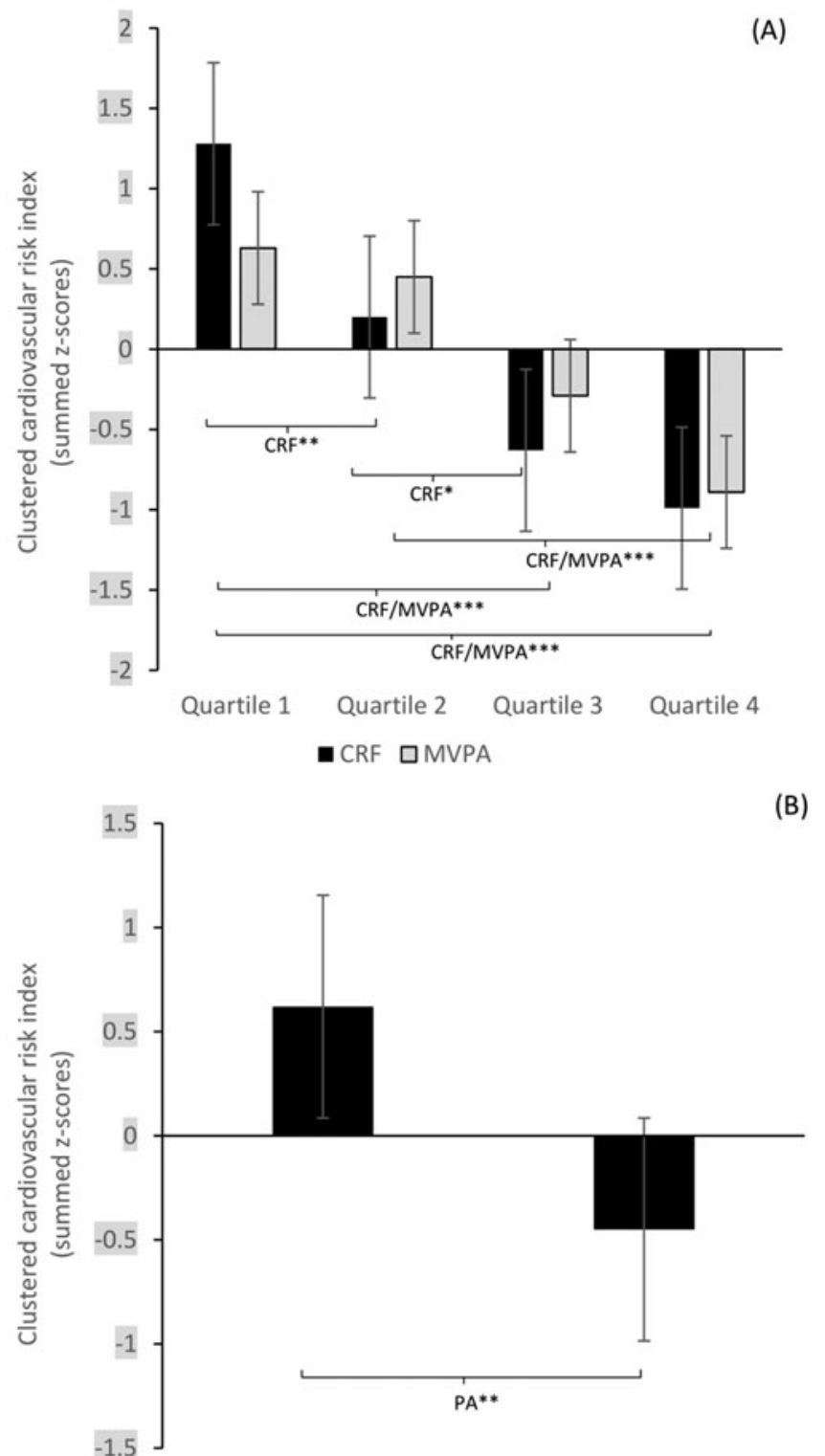

Not meeting PA recommendations

Meeting PA recommendations

Figure 1 Means for clustered metabolic risk, stratified by quartiles of cardiorespiratory fitness (CRF) and moderate-tovigorous physical activity (MVPA) (A) and by current international physical activity (PA) recommendations (B). Error bars represent SE. Data are adjusted for age, sex, socioeconomic status (SES) and ethnicity. ${ }^{\star} / * \star * \star *$ Differences are significantly different $\left({ }^{*} p<0.05,{ }^{* *} p<0.01,{ }^{* * *} p<0.001\right)$ between groups. If more than two groups are compared, group differences are based on Bonferroni posthoc tests.

cardiovascular risk score. Nevertheless, our findings are in line with those reported by Andersen and colleagues ${ }^{28}$ and Ekelund and colleagues, ${ }^{29}$ indicating that low levels of PA are linked to a clustering of cardiovascular disease risk factors in South African children, and particularly children living in socioeconomically deprived neighbourhoods. Moreover, our findings show that 4 out of 10 children do not meet recommended PA guidelines (at least $60 \mathrm{~min}$ of daily MVPA), with insufficient PA being particularly widespread among girls, black Africans and children aged 10-15 years. The low PA levels in our cohort of children from disadvantaged urban settings in townships of Port Elizabeth are not surprising given that options to perform PA are limited due to insecurity and poor school and public infrastructure. ${ }^{30}$ The conditions are compounded by a lack of basic services, including sewage and road infrastructure, overcrowding, violence, unemployment, large class sizes, lack of teachers with adequate physical education training and qualifications, lack of motivation and commitment, and a diverse set of learners and learning abilities in a single classroom. ${ }^{31}$ These contextual factors directly and indirectly influence children's health and well-being.

LMICs, including South Africa, are experiencing rapid urbanisation, which might have considerable negative effects on social PA inequalities in marginalised communities. ${ }^{32}$ In addition, policy change also plays a significant role in promoting this situation, as physical education in schools lost its stand-alone status and was marginalised in the South African national curriculum in 1994. Indeed, physical education was combined with other subjects in the 'Life Orientation' learning area. Thus, higher cardiovascular risks in children may be linked to the absence of institutionalised/ mandatory PA programmes in schools. Supplementary analyses indicated that children accumulated very little PA while at school. This raises the question of whether a single sport lesson per week is sufficient and whether such a physical education lesson is taught intensively enough for the individual learners. Earlier results obtained from the DASH project showed that higher CRF is associated with better cognitive performance in schoolchildren. $^{33}$

The following limitations should be considered when interpreting the key findings of our study. First, due to the cross-sectional nature of the data, we are unable to infer causality. Second, it is difficult to record all types of activities through waist-worn accelerometers, which might have led to an underestimation of PA levels. Third, due to natural fluctuations, a 7-day assessment period may not be fully representative of a child's 'typical' PA level and may still be too short and dependent on the climatic conditions during the assessment period. Fourth, the blood pressure results of the study subjects may be slightly increased due to experiencing an unknown situation.

\section{CONCLUSION}

Collectively, CRF and objectively assessed PA seem to be closely linked with a composite measure of children's cardiovascular risk. Given that 4 out of 10 South African schoolchildren do not meet international PA recommendations, efforts should be made to ensure that a physically active lifestyle is seen as an important educational goal. We believe that physical education at school can make an important contribution to children's overall PA levels. Hence, concerted actions are required for children in low-resourced 
communities in South Africa to (i) improve the status of, and resources allocated to, physical education in the South African school system and (ii) create conducive environments (eg, teacher training in physical education) that allow and stimulate regular PA in children, which meet the prognostic importance in childhood and adolescence of critical development periods for a healthy adult life.

\author{
Author affiliations \\ ${ }^{2}$ Department of Human Movement Science, Nelson Mandela University, Port \\ Elizabeth, South Africa \\ ${ }^{3}$ Novartis Foundation, Basel, Switzerland \\ ${ }^{4}$ Swiss Tropical and Public Health Institute, Basel, Switzerland \\ ${ }^{5}$ University of Basel, Basel, Switzerland \\ ${ }^{6}$ Novartis International AG, Basel, Switzerland
}

${ }^{1}$ Department of Sport, Exercise and Health, University of Basel, Basel, Switzerland

Acknowledgements The authors are grateful to the children and parents/guardians for their willingness to participate in the study. Special thanks are addressed to the education authorities of the participating schools, for facilitating support and collaboration of the teaching staff and the school health services. Moreover, we thank all the participating MSc and Honours students for their dedicated contribution to data collection.

Contributors IM, CW, RDR, HS, PS, NP-H, JU, UP and MG designed the study, established the methods and wrote the original study protocol. All other authors contributed to the development of the study protocol. IM, CW, RDR, LA, JD, SG, NJ, SN, DS, UP and MG conducted the fieldwork of the study. IM, LA, JD, SG, NJ, SN and DS managed data entry, cleaning and preparation of the database. Statistical analysis was done by MG. IM wrote the first draft of the manuscript with support of MG. CW, RDR, AA, LA, JD, SG, NJ, SN, SDR, DS, HS, PS, CW, NP-H, JU and UP provided comments on the drafts and have read and approved the final version of the manuscript prior to submission. IM and MG are guarantors of the paper.

Funding The cross-sectional study presented here was financially supported by the Novartis Foundation (Basel, Switzerland). The funder had no role in study design, data collection, data analysis or data interpretation, nor the decision to submit the paper for publication.

Competing interests The corresponding author had full access to all the data in the study and had final responsibility for the decision to submit for publication. All authors have completed the ICMJE uniform disclosure form at www.icmje.org/coi_disclosure. pdf, indicating no support from any other organisation for the submitted work, no financial relationships with any other organisations that might have an interest in the submitted work in the previous three years, and no other relationships or activities that could appear to have influenced the submitted work. AA and SDR are employees of Novartis Foundation (Basel, Switzerland); ChrW is an employee of Novartis International AG (Basel, Switzerland). All other authors declare no financial competing interests.

Ethics approval This study was approved by the ethics committees of Northwest and Central Switzerland (EKNZ; reference no. 2014-179, approval date: June 17, 2014), the Nelson Mandela University (NMU; study number H14-HEAHMS-002, approval date: July 4, 2014), the Eastern Cape Department of Education (approval date: August 3, 2014) and the Eastern Cape Department of Health (approval date: November 7, 2014). The study is registered at ISRCTN registry under controlled-trials.com (identifier: ISRCTN68411960, registration date: October 1, 2014).

Provenance and peer review Not commissioned; externally peer reviewed.

Data availability statement The raw datasets are available from the corresponding author on reasonable request.

Open access This is an open access article distributed in accordance with the Creative Commons Attribution Non Commercial (CC BY-NC 4.0) license, which permits others to distribute, remix, adapt, build upon this work non-commercially, and license their derivative works on different terms, provided the original work is properly cited, appropriate credit is given, any changes made indicated, and the use is non-commercial. See: http://creativecommons.org/licenses/by-nc/4.0/.

\section{ORCID iDs}

Ivan Müller http://orcid.org/0000-0002-6397-9979

Cheryl Walter http://orcid.org/0000-0001-8422-4105
Rosa Du Randt http://orcid.org/0000-0003-1033-9908

Ann Aerts http://orcid.org/0000-0003-1241-8730

Larissa Adams http://orcid.org/0000-0003-1459-1916

Jan Degen http://orcid.org/0000-0002-0644-3609

Stefanie Gall http://orcid.org/0000-0001-6617-4343

Nandi Joubert http://orcid.org/0000-0002-6920-981X

Siphesihle Nqweniso http://orcid.org/0000-0001-8793-9624

Danielle Smith http://orcid.org/0000-0002-7374-9034

Harald Seelig http://orcid.org/0000-0002-2178-4807

Peter Steinmann http://orcid.org/0000-0003-4800-3019

Christina Wadhwani http://orcid.org/0000-0001-5602-267X

Nicole Probst-Hensch http://orcid.org/0000-0001-8561-5976

Jürg Utzinger http://orcid.org/0000-0001-6885-0798

Uwe Pühse http://orcid.org/0000-0003-1228-316X

Markus Gerber http://orcid.org/0000-0001-6140-8948

\section{REFERENCES}

1 Guthold R, Stevens GA, Riley LM, et al. Worldwide trends in insufficient physical activity from 2001 to 2016 : a pooled analysis of 358 population-based surveys with 1.9 million participants. Lancet Glob Health 2018;6:e1077-e86.

2 Blüher M. Obesity: global epidemiology and pathogenesis. Nat Rev Endocrinol 2019;15:288-98.

3 WHO. Global recommendations on physical activity for health. Geneva: World Health Organization, 2010.

4 CDC. National Health and Nutrition Examination Survey (NHANES). Anthropometry procedures manual. 2011. Available https://www.cdc. gov/nchs/data/nhanes/nhanes_11_12/Anthropometry_Procedures_ Manual.pdf (accessed 16 Nov 2019)

5 CDC. How much physical activity do children need? Atlanta, GA, USA: Centers for Disease Control and Prevention, 2017.

6 The Lancet. Join the Lancet 2020 campaign on child and adolescent health. Lancet 2020;395:89.

7 American College of Sports Medicine. Physical fitness in children and youth. Med Sci Sports Exerc 1988;20:422-3.

8 Strong WB, Malina RM, Blimkie CJ, et al. Evidence based physical activity for school-age youth. J Pediatr 2005;146:732-7.

9 McVeigh JA, Norris SA, de Wet T. The relationship between socio-economic status and physical activity patterns in South African children. Acta Paediatr 2004;93:982-8.

10 Draper CE, Tomaz SA, Jones RA, et al. Cross-sectional associations of physical activity and gross motor proficiency with adiposity in South African children of pre-school age. Public Health Nutr 2018;22:614-23.

11 Amnesty International. Broken and unequal. The state of education in South Africa. London: Amnesty International, 2020.

12 Draper CE, Tomaz SA, Bassett SH, et al. Results from South Africa's 2018 report card on physical activity for children and youth. J Phys Act Health 2018;15:S406-08.

13 Andersen LB, Wedderkopp N, Hansen HS, et al. Biological cardiovascular risk factors cluster in Danish children and adolescents: the European youth heart study. Prev Med 2003;37:363-7.

14 National Treasury of South Africa. Intergovernmental Fiscal Reviews (IGFR): education 2015. Available www.treasury.gov.za (accessed 21 Nov 2019)

15 Yap P, Müller I, Walter C, et al. Disease, Activity and Schoolchildren's Health (DASH) in Port Elizabeth, South Africa: a study protocol. BMC Public Health 2015;15:1285.

16 Slaughter MH, Lohman TG, Boileau RA, et al. Skinfold equations for estimation of body fatness in children and youth. Hum Biol 1988;60:709-23. https://digitalcommons.wayne.edu/humbiol/vol60/ iss $5 / 4 /$

17 Neelam D, Diepart M, Dziekan G, et al. WHO. WHO guidelines on drawing blood: best practices in phlebotomy. Geneva: World Health Organization, 2010. https://www.euro.who.int/_data/ assets/pdf file/0005/268790/WHO-guidelines-on-drawing-bloodbest-practices-in-phlebotomy-Eng.pdf?ua $=1$ ISBN: 9789241599221

18 Parikh $\mathrm{P}$, Mochari $\mathrm{H}$, Mosca L. Clinical utility of a fingerstick technology to identify individuals with abnormal blood lipids and high-sensitivity C-reactive protein levels. Am J Health Promot 2009:23:279-82.

19 Hills AP, Mokhtar N, Byrne NM. Assessment of physical activity and energy expenditure: an overview of objective measures. Front Nutr 2014;1:5.

20 Troiano RP, Berrigan D, Dodd KW, et al. Physical activity in the United States measured by accelerometer. Med Sci Sports Exerc $2008 ; 40: 181-8$ 
21 Clemente FM, Nikolaidis PT, Martins FM, et al. Physical activity patterns in university students: do they follow the public health guidelines? PLoS One 2016;11:e0152516.

22 Aadland E, Andersen LB, Skrede T, et al. Reproducibility of objectively measured physical activity and sedentary time over two seasons in children; comparing a day-by-day and a week-by-week approach. PLoS One 2017;12:e0189304.

23 Evenson KR, Catellier DJ, Gill K, et al. Calibration of two objective measures of physical activity for children. J Sports Sci 2008;26:1557-65.

24 Léger L, Lambert J, Goulet A, et al. Capacité aérobie des Québécois de 6 à 17 ans - test navette de 20 mètres avec paliers de 1 minute. Can J App/ Sport Sci 1984;9:64-9. https://www.semanticscholar.org/ paper/Capacit\%C3\%A9-a\%C3\%A9robie-des-Qu\%C3\%A9b\%C3\% A9cois-de-6-\%C3\%A0-17-ans-test-L\%C3\%A9ger-Lambert/ 7e5d1040bc94b1b546e179f9cc5c0bafc8e77a52

25 Léger LA, Mercier D, Gadoury C, et al. The multistage 20 meter shuttle run test for aerobic fitness. J Sports Sci 1988;6:93-101.

26 Armstrong NJ, Williams J, Ringham D. Peak oxygen uptake and progressive shuttle run performance in boys aged 11 to 14 years. Brit J Phys Ed 1988;19:10-1.

27 West SG, Finch JF, Curran PJ. Structural equation models with nonnormal variables: problems and remedies. In: Hoyle $\mathrm{RH}$, ed
Structural equation modeling concepts, issues, and applications. Thousand Oakes: Sage, 1995: 56-75.

28 Andersen LB, Harro M, Sardinha LB, et al. Physical activity and clustered cardiovascular risk in children: a cross-sectional study (the European Youth Heart Study). Lancet 2006;368:299-304.

29 Ekelund U, Anderssen SA, Froberg K, et al. Independent associations of physical activity and cardiorespiratory fitness with metabolic risk factors in children: the European Youth Heart Study. Diabetologia 2007;50:1832-40.

30 Zulu BM, Urbani G, van der Merwe A. Violence as an impediment to a culture of teaching and learning in some South African schools. South Afr J Educ 2004;24:170-5. https://www.ajol.info/index.php/saje/arti cle/view/24984

31 Rajput D, Van Deventer K. An epoch of controversy within physical education and sport in post-apartheid South Africa: a review. Afr J Phys Health Educ Recreation Dance 2010;16:147-65.

32 Van Cauwenberg J, De Clercq B, Deforche B, et al. Accuracy and inequalities in physical activity research. Lancet Glob Health 2019;7: e183-e84.

33 Gall S, Adams L, Joubert N, et al. Effect of a 20-week physical activity intervention on selective attention and academic performance in children living in disadvantaged neighborhoods: a cluster randomized control trial. PLoS One 2018;13:e0206908. 